\title{
Editorial
}

\section{The Circumcision Issue}

David A. Bloom, MD

Harry P. Koo, MD

\section{The Debate}

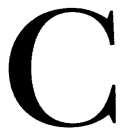

ircumcision of the male prepuce, as a purposeful surgical intervention, is nearly as old as the human species and is firmly rooted in many cultures and religions. There must have been some pervasive reasons for its durability. Yet even two millennia past, cosmetic aspects of circumcision were challenged when, for example, Celsus proposed an operation to restore the prepuce. ${ }^{1}$ The pros and cons of male circumcision still provoke contentious and inflammatory debate. Thoughtful scientific studies and vigorous discussion in letters to editors recur in major medical journals. ${ }^{2,3}$ The debate is permeated by science and opinion, superstition and pseudoscience, culture and religion. Into the fray are the issues of urinary infection susceptibility, sexually transmitted diseases, penile cancer, cervical cancer, genital sensitivity, sexual performance, and emotional impact. ${ }^{4}$

\section{The Polar Extremities of the Debate}

Some males will need a circumcision in their lives, most likely for true phimosis or recurrent posthitis. Debaters might claim that a retraction program, dorsal slit, or preputial plasty ${ }^{5}$ should suffice, but our point is simply that some males will need something done for a foreskin problem in their lifetimes and the solution is most likely to be circumcision. On the other hand, some male infants should not be circumcised: these are boys with hypospadias or significant isolated chordee; their foreskin will be an asset in later reconstruction.

For most males, in between these polar situations, we think that the debate is not an important medical issue but rather a matter of family choice. This is not to deny that individual medical circumstances may play into a decision to circumcise. For example, a boy with vesicoureteral reflux may benefit from one

Clin Pediatr. 1999;38:243-244

From the University of Michigan, Ann Arbor, Michigan.

Reprint requests and correspondence to: David A. Bloom, MD, Professor of Surgery, University of Michigan, 1500 East Medical Center Drive, Ann Arbor, MI 48109-0330.

(C) 1999 Westminster Publications, Inc., 708 Glen Cove Avenue, Glen Head, NY 11545, U.S.A.

less infection-facilitative factor, or a severely handicapped child may not be able to maintain adequate genital hygiene. For most male infants today no robust scientific evidence proves one path is better. In the absence of valid data, any decision, in essence, is heuristic ${ }^{6}$ and is best made by each family with information and guidance (not bias) from the physician.

\section{Can Science Answer the Debate?}

Science, Edward O. Wilson writes, "is the organized enterprise that gathers knowledge about the world and condenses the knowledge into testable laws and principles." 7 The critical features that distinguish science from pseudoscience, he shows, are repeatability, economy, mensuration, heuristics, and consilience. An Orwellian world could scientifically solve the preputial debate by taking two large matched cohorts at birth and circumcising one and not circumcising the other. The families of the uncircumcised and later the boys themselves would be given instructions on care of the prepuce, but it is reasonable to assume that some of this cohort would need late circumcision (or some treatment) for preputial problems. 
The circumcised group would be managed with the best methodology available, but it is still likely that a small percentage would have early and late complications from the procedure. Each cohort would be followed up for life. Critical parameters would be recorded, especially costs, complications, secondary procedures, psychological profiles, and sexual function. We suspect the two cohorts, in this imaginary study, would end up closely matched on final analysis. But that is only our suspicion. Lacking such a study we remain subject to data from more limited studies, opinion, pseudoscience, cultural trends, and superstition.

\section{Who Performs Circumcisions?}

This question needs to be divided into two questions, and each will have a different answer. For neonatal circumcisions the answer will typically be obstetricians, pediatricians, family practitioners, and religious figures. For postnatal circumcisions the answer will be urologists, pediatric surgeons or general surgeons. Incongruously, neonatal circumcision is performed by those without expertise in genitourinary function and reconstruction. Nevertheless, most pediatric urologists see only occasional problems from such procedures, suggesting that they usually turn out well.

\section{Methods, Analgesia, Complications and Caveat}

Once the pro-and-con debate has been addressed, the major remaining issues with neonatal circumcision are methodology, pain, and complication. The Gomco ${ }^{\circledR}$ clamp, the Mogen ${ }^{\circledR}$ clamp, excision by mohel, ${ }^{8}$ or Plastibell ${ }^{\circledR}$ methods have their advocates. ${ }^{9}$ Of course circumcision hurts babies; when skin is cut or crushed, mammals usually feel pain. There is no reason or evidence to expect that the human neonatal prepuce is devoid of sensation. We have tools to minimize discomfort and suffering, so why should the issue of pain even be in question? ${ }^{10-12}$ Circumcision complications relate to experience and technique. Plastibell ${ }^{\circledR}$ complications are addressed by Holmes, Canning, and Cilento in this issue of the Journal. ${ }^{13}$ These authors discuss the safety steps, pitfalls, and risks of this popular and simple method. Their points are important and clearly expressed. One other complication to point out is meatal stenosis. ${ }^{14}$ This long-term problem is most likely due to irritation of the delicate unprotected meatal lips, chronically rubbing against diapers. A dab of clear protective ointment with each diaper change, particularly in the first several months after circumcision, may prevent significant meatal stenosis later in childhood.

Any provider who would undertake neonatal circumcision should offer information, discussion, and alternatives to families. Personal bias should be left at home. When the prepuce is left intact, instructions should be given regarding care of the foreskin. Families should learn the signs of posthitis and paraphimosis. Clinicians should avoid forcible retraction or any manipulation in the clinic that can injure the prepuce. If circumcision is selected, the provider should be knowledgeable regarding methods and well trained and skilled in at least one technique plus anesthesia. Complications should be understood and recognized, with a well-planned means of redress if that should be necessary. Lastly, the outcome must be assessed in the longitudinal care of a child all the way through toilet training and adolescence.

\section{REFERENCES}

1. Rubin JP. Celsus' decircumcision operation. Urology. 1980;16:121-124.

2. Wiswell TE. Circumcision circumspection. N Engl J Med. 1997;336:12441245.

3. Laumann EO, Masi CM, Zuckerman EW. In reply (letter). JAMA. 1997; 278:203.

4. Cromie WJ, Kelleher RE. Circumcision. JAMA. 1993;269:529-530.

5. Cuckow PM, Rix G, Mouriquand PDE. Preputial plasty: a good alternative to circumcision. J Pediatr Surg. 1994;29: 561-563.

6. McDonald CJ. Medical heuristics: the silent adjudicators of clinical practice. Ann Intern Med. 1996;124:56-62.

7. Wilson EO. Consilience. The Unity of Knowledge. New York: Alfred A. Knopf, Inc.; 1998:53.

8. Shechet J, Fried SM, Tannenbaum B. To the editor (letter). JAMA. 1998; 279:1170.

9. Kunin SA. Circumcision in newborns: not why, but how. Dialogues Pediatr Urol. 1994;17:1-8.

10. Ryan CA, Finer NN. Changing attitudes and practices regarding local analgesia for newborn circumcision. Pediatrics. 1994;94:230-233.

11. Horger EO III, Arnett RM, Jones JS. Local anesthesia for infants undergoing circumcision. JAMA. 1998;279: 1169.

12. Lander J, Brady-Fryer B, Metcalfe JB, et al. Comparison of ring block, dorsal penile nerve block, and topical anesthesia for neonatal circumcision. JAMA. 1997;278:2157-2162.

13. Cilento BG Jr, Holmes NM, Canning DA. Plastibell ${ }^{\circledR}$ complications revisited. Clinical Pediatrics 1999;4:239-242.

14. Persad R, Sharma S, Mc Tavish J, et al. Clinical presentation and pathophysiology of meatal stenosis following circumcision. Br J Urol. 1995;75:91-93. 